\title{
9. Diagnosis of Cerebrovascular Disease by Echoencephalogram, Electroencephalogram and Regional Cerebral Blood Flow
}

\author{
H. Nagai, K. Sakurai, M. Hayashi, M. Furuse, K. Okamura, \\ A. Shintani, Y. OKa, T. Kobayashi and A. Ikeyama \\ 2nd Department of Surgery, School of Medicine, Nagoya University
}

In order to improve the results of neurosurgical operation for intracerebral hemorrhage, it is the most important to determine accurately what sort of patients with acute stroke must be taken to operation.

As a cerebral apoplexy includes many different pathognomic diseases (i.e. hemorrhage and infarction etc.), it is a different problem to differentiate intracerebral hemorrhage from the other apoplectic diseases by means of only neurological methods.

However, the recent development of echoencephalography (echo-EG) and electroencephalography seems to us quite useful in the differential diagnosis of acute cerebrovascular diseases.

The purpose of the present paper is to know the usefulness of echo-EG and EEG by diagnosing the patients with severe cerebral apoplexy.

\section{1) Echoencephalogram}

a) Experimental results

Fig. 1 showed echo-EG of intracerebral hematoma at experimental intervals after the infusion of clot. One day after the infusion of clot, multiple and sharp echoes were observed corresponding to the localization and the size of hematoma, and also the displacement of midline echo was observed. After 3 to 7 days, the hematoma echoes became less prominent and weakened following the experimental days. Histologically, after 3 to 7 days, the boundary of hematoma became less distinct than that of the first day, and the content of hematoma became homogenous due to autolysis. As varying of content of hematoma, echo-EG findings were of different results. That is, only when the clot was infused, we found multiple and sharp echoes as above mentioned. However, whole blood added with heparin or liquified blood (whole blood one part and Ringer solution three parts) revealed only a single or two phasic echo. (Fig. 2) From above mentioned results, it is reasonable to presume that the multiple and sharp echoes (so-called hematoma echo) only found in the case of the hematoma having the clot.

Otherwhile, there were no characteristic echos through the intervals, although a distinct difference was histologically observed between the normal brain tissue and the area of softening. 


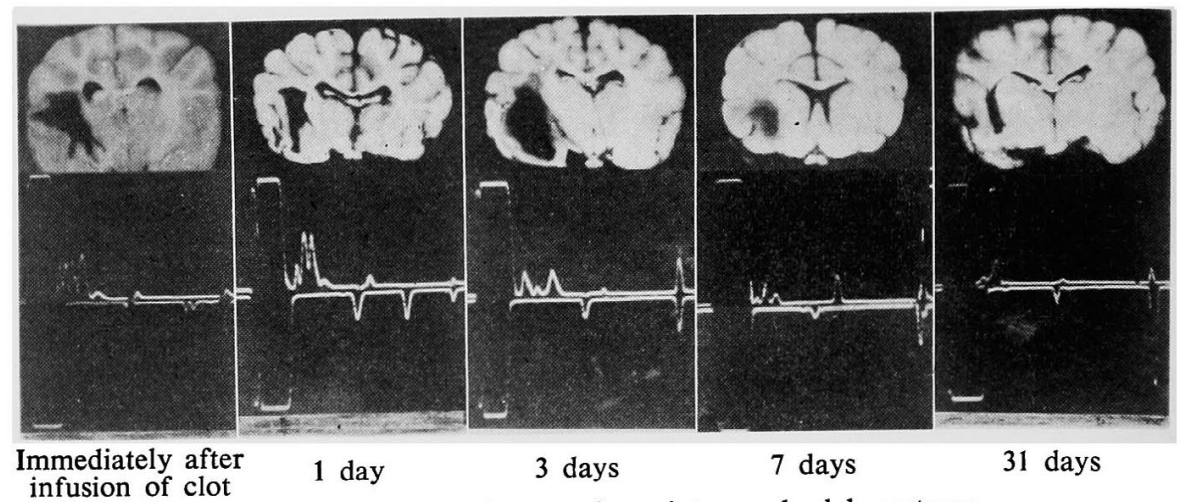

Fig. 1. Echoencephalogram from intracerebral hematoma.

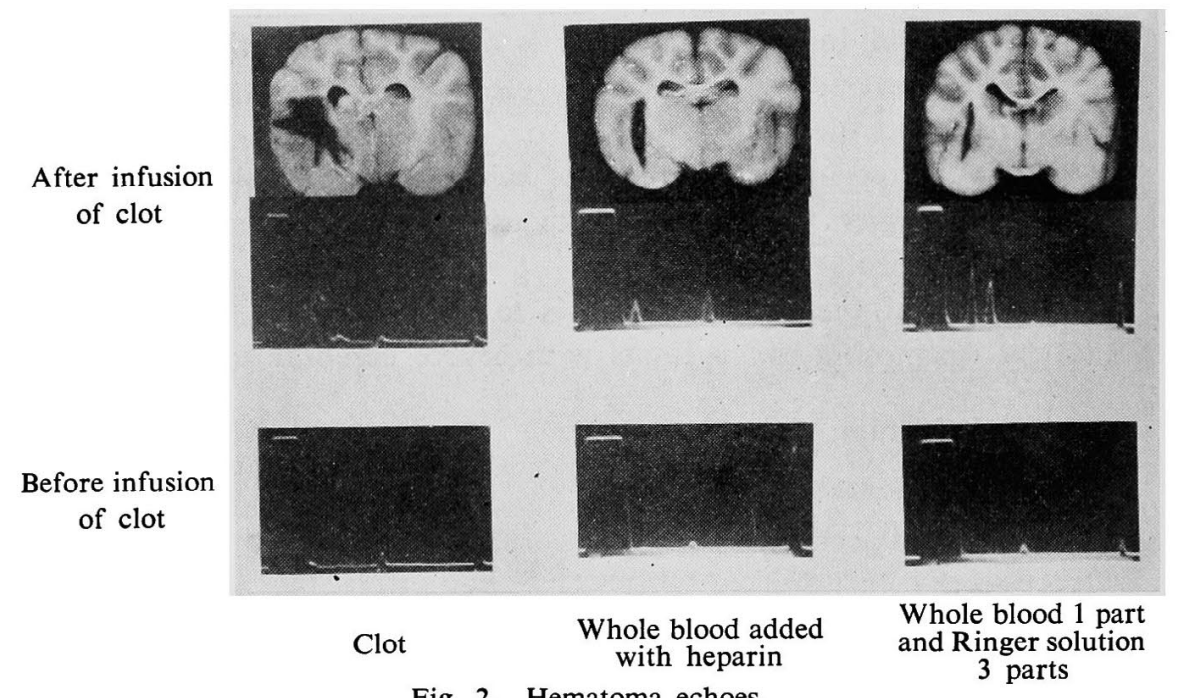

Fig. 2. Hematoma echoes.

b) Clinical results

We also investigated echo-EG of clinical cases with acute cerebrovascular diseases; 7 intracerebral hemorrhage and 13 cerebral infarction. In the case of hemorrhage, 4 cases showed multiple echoes and shift of midline echo, 2 cases only shift of midline echo, and the other case no findings. On the contrary, there were no specific ecnoes in the 13 cases of cerebral infarction. Therefore, it is strongly suggested that the clinical use of echo-EG for diagnosis of early cerebral hemorrhage and infarction is not only possible, but also very practical.

2) EEG and regional cerebral bood flow (CBFr)

a) Experimental results

In the serial EEG investigation, the tendency of gradual restoration 


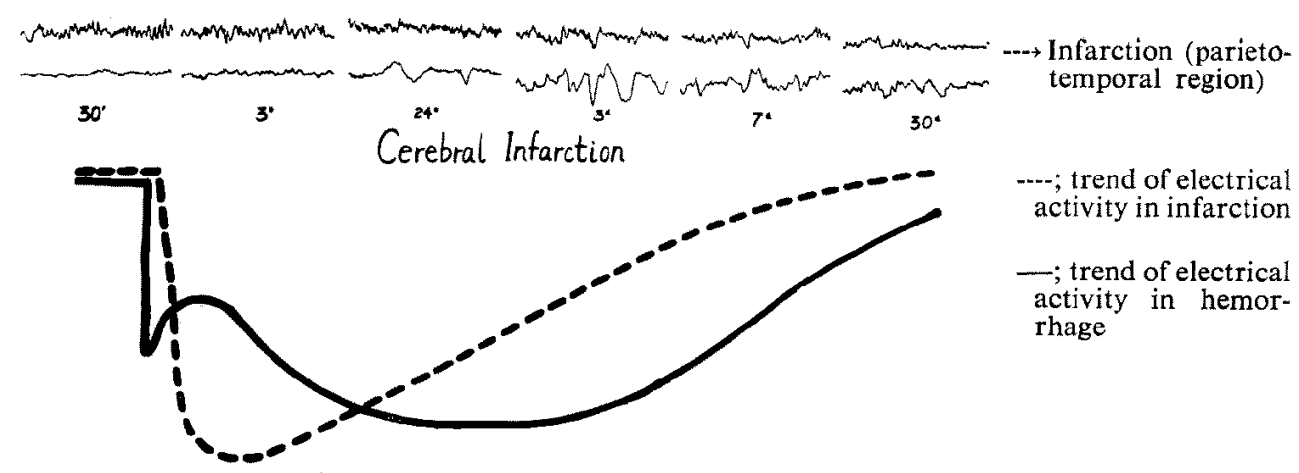

\section{Intracerebral Hematoma}

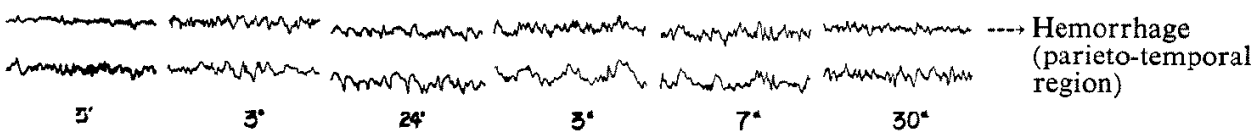

Fig. 3. Serial EEG records in dogs.
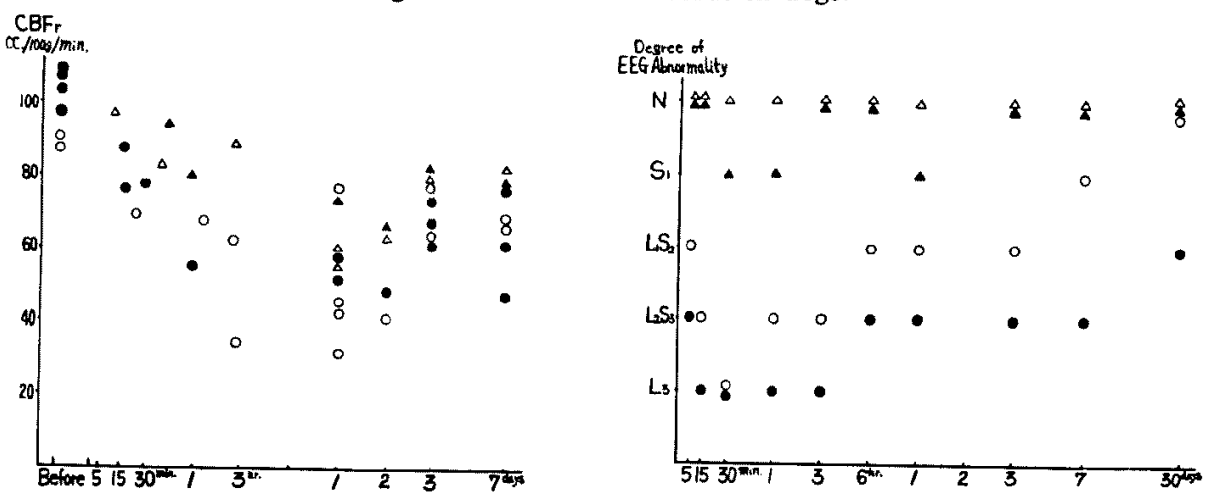

Fig. 4. Serial EEG changes (right) and CBFr (left) in cerebral infarction.
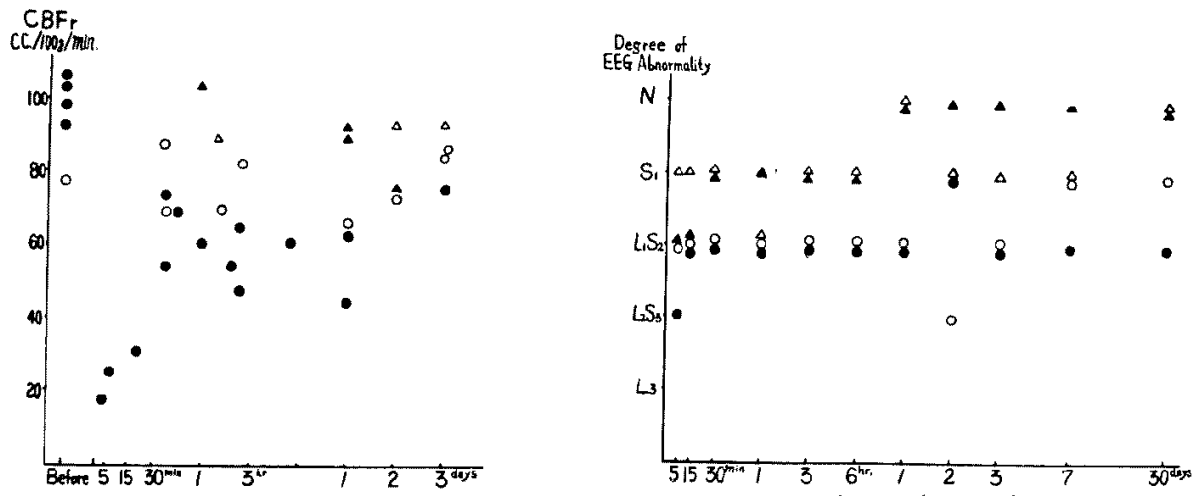

$\mathrm{N}$; normal or borderline, $\mathrm{S}_{1}$; slight slow activity, $\mathrm{L}_{1} \mathrm{~S}_{2}$; moderate low voltage or moderate slow activity, $L_{2} S_{3}$; severe low voltage or severe slow activity, $L_{3}$; extremely severe low voltage (flattening),

$O$; ipsilateral froantl area

$\triangle$; contralateral frontal area

- ; ipsilateral parietotemporal area

A; contralateral parietotemporal area

Fig. 5. Serial EEG changes (right) and CBFr (left) in intracerebral hematoma. 
was observed after the onset in the case of brain softening, with negligible changes in the contralateral hemisphere. On the other hand, the temporary deterioration for 3 to 7 postoperative days, followed by progressive improve. ment in the cases with intracerebral hemorrhage. Comparing with the results of cerebral in farction, contralateral EEG changes in the later cases were significant, particularly in early stage. (Fig. 3)

In this paper we intended to correlate these EEG findings with value of CBFr determined by Krypton 85 (Ingver's method).

The value of CBFr showed rapid fall immediately after the ligation of the middle cerebral artery, especially in the parieto-temporal region on the ipsilateral side. Succeeding improvement of $\mathrm{CBFr}$ was gradual and lineal. On the contralateral side, $C B F r$ remained within the normal range of variation. These CBFr findings were parallel to EEG findings in the cases of cerebral infarction (Fig. 4)

On the other hand, after the infusion of clot, the value of $\mathrm{CBFr}$ decreased gradually and showed the lowest level between 3 hrs and 3 days. In the same period, there was moderate decrease of $C B F r$ in the contralateral hemisphere. These CBFr findings also parallel to EEG findings in the cases of intercerebral hemorrhage. (Fig. 5)

b) Clinical results

i) Extensive cerebral infarction (4 cases)

EEG of these patients showed ipsilateral depression of voltage in early clinical stage and was gradually followed by slow activity. On the contralateral side, the background activity was relatively well reserved.

ii) Intracerebral hemorrhage (5 cases)

Shortly after the stroke, EEG showed abnormalities with a varying degree in both hemispheres, particularly on the affected side. After removal of the hematoma, these changes were distinctively reduced, particularly in the contralateral hemisphere, while remained in relatively localized region on the ipsilateral side.

iii) Fatal case

In a case with massive intraventricular hemorrhage, severe suppression of the electrical activity was observed over both hemispheres and deteriorated gradually in serial EEG records. The deteriorating trend seems to be indicating poor prognosis. 